\title{
Core curriculum for medical physicists in radiology. Recommendations from an EFOMP/ESR working group
}

\author{
Jacob Geleijns • Éamann Breatnach • \\ Alfonso Calzado Cantera • John Damilakis • \\ Philip Dendy • Anthony Evans • Keith Faulkner • \\ Renato Padovani • Wil Van Der Putten • Lothar Schad • \\ Ronnie Wirestam • Teresa Eudaldo • \\ on behalf of the European Federation of Organisations \\ for Medical Physics (EFOMP) and the European Society \\ of Radiology (ESR)
}

Received: 21 March 2012 / Accepted: 21 March 2012 /Published online: 3 May 2012

(C) European Society of Radiology 2012

\begin{abstract}
Some years ago it was decided that a European curriculum should be developed for medical physicists professionally engaged in the support of clinical diagnostic imaging departments. With this in mind, EFOMP (European Federation of Organisations for Medical Physics) in association with ESR (European Society of Radiology) nominated an expert working group. This curriculum is now to hand.
\end{abstract}

\section{J. Geleijns $(\bowtie)$}

Leiden University Medical Center,

Albinusdreef 2,

2333 ZA Leiden, The Netherlands

e-mail: K.Geleijns@lumc.nl

É. Breatnach

Mater Misericordiae University Hospital,

Dublin, Ireland

\section{A. C. Cantera}

Complutense University of Madrid,

Madrid, Spain

J. Damilakis

University of Crete,

Heraklion, Greece

P. Dendy

Addenbrookes NHS Trust,

Cambridge, United Kingdom

\section{A. Evans}

University of Leeds,

Leeds, United Kingdom

K. Faulkner

Quality Assurance Reference Centre,

Wallsend, United Kingdom
The curriculum is intended to promote best patient care in radiology departments through the harmonization of education and training of medical physicists to a high standard in diagnostic radiology. It is recommended that a medical physicist working in a radiology department should have an advanced level of professional expertise in X-ray imaging, and additionally, depending on local availability, should

\section{R. Padovani}

S. Maria Hospital,

Udine, Italy

W. Van Der Putten

University Hospital Galway,

Galway, Ireland

L. Schad

Faculty of Medicine Mannheim, University of Heidelberg, Mannheim, Germany

R. Wirestam

Lund University Hospital,

Lund, Sweden

T. Eudaldo

Santa Creu i Sant Pau Hospital,

Barcelona, Spain 
acquire knowledge and competencies in overseeing ultrasound imaging, nuclear medicine, and MRI technology. By demonstrating training to a standardized curriculum, medical physicists throughout Europe will enhance their mobility, while maintaining local high standards of medical physics expertise. This document also provides the basis for improved implementation of articles in the European medical exposure directives related to the medical physics expert. The curriculum is divided into three main sections: The first deals with general competencies in the principles of medical physics. The second section describes specific knowledge and skills required for a medical physicist (medical physics expert) to operate clinically in a department of diagnostic radiology. The final section outlines research skills that are also considered to be necessary and appropriate competencies in a career as medical physicist.

Keywords Medical physics · Radiology $\cdot$ Education and training $\cdot$ Curriculum

\section{Introduction}

The medical physicist in radiology is essential for enabling the practice of safe, state-of-the-art medical imaging. Medical physicists in radiology are members of the multidisciplinary clinical teams that are responsible for radiology services to patients. Their role is to provide critical scientific input on the physical processes and technology that underpin the whole radiology service. Generally, the medical physicists in radiology design and develop the framework of medical imaging, image processing, image distribution, image storage, radiation dosimetry, quality assurance of the imaging equipment, information and communication technology (ICT) aspects of the imaging process, and radiation protection of the patient and operator. Specifically, the medical physicists in radiology provide expert advice on the development, implementation, and improvement of imaging techniques and processes. They also provide expert input for applications of medical imaging outside the radiology department. Medical physicists in radiology have a leading role in the strategic planning, testing, safe use, and optimization of advances in medical imaging technologies and techniques.

Both initial and continuing education and training are necessary in order to acquire and maintain sufficient knowledge and an appropriate level of knowledge and competence. The objective of our work was to develop a core curriculum for the education and training of European medical physicists in radiology. This core curriculum for medical physicists in radiology is issued jointly by the European Federation of Organisations for Medical Physics (EFOMP) and the European Society of Radiology (ESR). It provides guidelines for the education and training of medical physicists in medical imaging. These recommendations accommodate the contemporary requirements for the knowledge and competency needs in this rapidly evolving field of medicine.

\section{Materials and methods}

In 2004, a European expert group published the first European core curriculum for medical physicists in radiotherapy [1], and they recently finalized a revised and updated version of this curriculum [2]. The curriculum for medical physics in radiotherapy was developed and revised jointly by EFOMP and the European Society for Radiotherapy and Oncology (ESTRO). Another expert group for the development of a European core curriculum for medical physicists in radiology was initiated and installed by the EFOMP with participation of ESR. The radiology medical physics group represents experts in general projection radiography, mammography, diagnostic fluoroscopy, interventional radiology, computed tomography, magnetic resonance imaging, ultrasound imaging, and radiation protection.

The basis for a draft curriculum on radiology medical physics was laid during meetings of the expert group, and in a later phase written feedback from the experts was used to finalize the curriculum. It was decided to develop a curriculum that was consistent in structure and format with the revised radiotherapy medical physics curriculum.

It was also decided that the curriculum should define general competencies; specific knowledge, skills, and competencies in medical physics; and a research project.

\section{Results}

A curriculum for the education and training of medical physicists in radiology was established [3]. The medical physicist in radiology must be able to work together with the multi-disciplinary team in the clinical application of medical imaging and participate in the organizing and structuring of the medical imaging process. The physicist should also ensure that the well-being, interests, and dignity of patients are promoted and safeguarded at all times.

To support these requirements, the first part of the curriculum presents general competencies, subdivided into two sections: organization and professionalism. The second part defines specific knowledge, skills, and competencies in medical physics required to operate clinically in a department of radiology. This part is subdivided into the sections fundamental knowledge and applied knowledge. A medical physicist requires clinical and analytical skills and is also expected to remain abreast of developments in medical imaging. Both 
aspects of the job can be trained through the development of research skills. These need not necessarily be acquired through abstract academic research but can equally likely be developed through applied clinical problems. Thus, the third and final part of the curriculum describes a research project where these skills are developed. Table 1 gives an overview of the structure of the curriculum.

\section{Discussion}

The European core curriculum for medical physicists in radiology can be used when national curricula are being developed. The core curriculum supports the harmonization of education and training of European medical physicists in radiology, it may help to improve their mobility within Europe, and it provides the basis for improved implementation of articles in European medical exposures directives that are related to the medical physics expert. The core curriculum aims at bringing the medical physicist in radiology up to the level of a qualified medical physicist. A qualified medical physicist is an individual who is competent to practice independently and to register as a medical physicist, in one or more of the subfields of medical physics. To act as an expert, further experience is required, and an involvement in a program for continuing professional development is highly recommended.

Radiology involves many subspecialties including radiography, mammography, computed tomography, X-ray guided interventions, and pediatric radiology but also magnetic resonance imaging (MRI) and ultrasound imaging. The medical physicist in radiology who is recognized as a medical physics expert must have a high level of expertise in Xray imaging. In addition, but depending on local conditions, it may also be desirable or even required that the medical physicist in radiology acquires a certain level of expertise in MRI, ultrasound imaging, and nuclear medicine. Consequently, a trainee is not expected to cover all elements of this curriculum. The curriculum can be downloaded from: www.efomp.org and www.myesr.org.
Table 1 The structure of the core curriculum for medical physicists in radiology

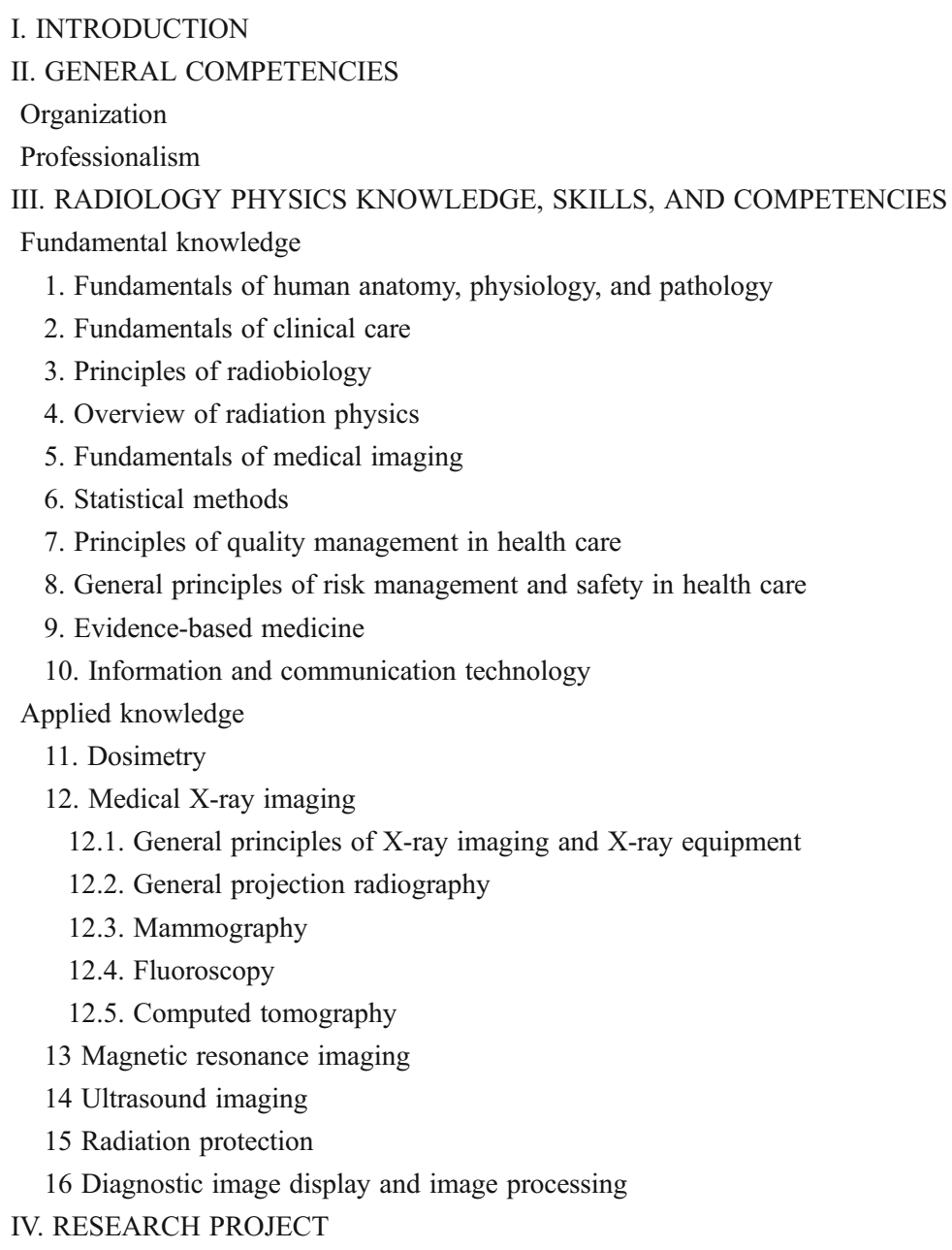




\section{References}

1. Eudaldo T, Huizenga H, Lamm IL et al (2004) Guidelines for education and training of medical physicists in radiotherapy. Recommendations from an ESTRO/EFOMP working group. Radiother Oncol 70:125-135
2. Olsen DR, Beavis A, Eudaldo T, et al. (2011) Recommended ESTRO core curriculum for medical physicists working in radiotherapy, 2nd edition. Radiother Oncol 2011 (in press)

3. EFOMP, ESR (2012) Core curriculum for medical physicists in radiology. EFOMP website: http://www.efomp.org/professionalissues/policy-statements.html ESR website:http://www.myesr.org/ $\mathrm{cms} /$ website.php?id=/en/education_training/further_curricula.htm 